hep-ph/9608323

IOA.02/96

NTUA 55-96

\title{
Duality Constraints on Supersymmetric Unified Models and Radiative Symmetry Breaking ${ }^{\dagger}$
}

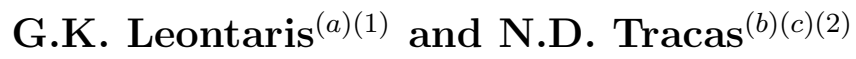 \\ (a) Theoretical Physics Division, Ioannina University \\ GR-451 10 Ioannina, Greece \\ (b) Physics Department, National Technical University \\ GR-15780 Zografou, Athens, Greece \\ (c) Theory Division, CERN \\ CH-1211 Geneva 23, Switzerland
}

\begin{abstract}
Motivated from unified models with string origin, we analyse the constraints from duality invariance on effective supergravity models with intermediate gauge symmetry. Requiring vanishing vacuum energy and invariance of the superpotential couplings, the modular weights are subject to various constraints. Further, the intermediate gauge symmetry breaking scale $M_{U}$ is related to the values of modular weights of the matter and higgs fields. For certain regions of values of the latter, $M_{U}$ can be close to the conventional unification scale of the minimal supersymmetric standard model. We also examine particular examples where the intermediate gauge symmetry breaks down to the standard gauge group radiatively.
\end{abstract}

IOA $02 / 96$

NTUA 55-96

August 1996

†Work partially supported by C.E.C. Projects SC1-CT91-0729 and CHRX-CT93-0319.

(1) leonta@cc.uoi.gr

${ }^{(2)}$ ntrac@central.ntua.gr 
If one adopts the idea for unification of all forces, the minimal supersymmetric standard model (MSSM) is considered as the most natural extension of the standard model (SM) of strong and electroweak interactions, since its spectrum allows the three gauge couplings to meet at an energy of $\mathcal{O}\left(10^{16} \mathrm{GeV}\right)$ [1]. Besides, unified supersymmetric models solve successfully the hierarchy problem [2] of their corresponding nonsupersymmetric versions. Yet, the MSSM has many arbitrary parameters and by no means can be considered as the ultimate theory of elementary particles. The only road beyond the MSSM which looks promising these days is $N=1$ supergravity [3] coupled to matter and gauge fields. However, $N=1$ supergravity still contains a lot of arbitrariness. One is free to choose the chiral multiplets provided they transform consistently under the chosen gauge group, while Yukawa couplings in the superpotential are also arbitrary. Nowadays, string theory appears as the only serious candidate which could predict all the above arbitrary parameters. On the other hand, strings can have as a limit an effective $N=1$ supergravity theory. In addition, the gauge group of most of the string constructions, contrary to what was usually assumed in the old Grand Unified approach [ 沟, is predicted to have a product structure, rather than being a single gauge group. Thus, if string theory really plays a role in particle physics, then one is left with an effective theory with the following summarized general characteristics. There is an effective unification scale, namely the string scale $M_{s t r}$, where all couplings - up to threshold corrections - attain a common value. At this point one is left with an effective $N=1$ supergravity theory while the gauge group structure is of the form $G=\prod_{n} G_{n}$, which usually contains an 'observable' and a 'hidden' part. In general, the observable part has a rank larger than that of the MSSM symmetry. In such a case, we may say that $G$ is an Intermediate Gauge Symmetry (IGS) which breaks down to the SM - gauge group at an intermediate scale $M_{X}$, usually some two orders of magnitude below the string scale. Moreover, string symmetries give rather strong constraints on many of the parameters of the effective field theory model. For example, the kinetic term appears to have a certain structure, while the effective Lagrangian is in general invariant under certain duality symmetries which act on the space of the moduli [5]. The superpotential and the Yukawa couplings is also subject to similar constraints. In the present work, we would like to present an analysis of the modular invariance constraints in effective models arising from the string. In particular, we have in mind models with an IGS scale which often appear in string constructions. We will examine the following issues. Using the constraints of modular invariance we will determine the properties of the effective potential in IGS models. Requiring also zero cosmological constant, we will correlate the vacuum expectation values of the higgs fields breaking the IGS group, with the modular weights. We will finally present a particular example where gauge symmetry may break radiatively.

In $N=1$ supergravity one introduces a real gauge invariant Kähler function whose 
general form is [6]

$$
G(z, \bar{z})=\mathcal{K}(z, \bar{z})+\log |\mathcal{W}(z)|^{2}
$$

where $\mathcal{K}(z, \bar{z})$ is the Kähler potential whose second derivatives determine the kinetic terms for the various fields in the chiral supermultiplets (we are using the standard supergravity mass units). $\mathcal{W}$ is the superpotential which is a holomorphic function of the chiral superfields. Denoting $z=(\Phi, Q)$, where $\Phi$ stands for the dilaton field $\mathrm{S}$ and other moduli $T_{i}$ while $\mathrm{Q}$ for the chiral superfields, we may expand the Kähler potential as follows

$$
\mathcal{K}(\Phi, \bar{\Phi}, Q, \bar{Q})=K(\Phi, \bar{\Phi})+Z_{\bar{i} j}(\Phi, \bar{\Phi}) \bar{Q}_{\bar{i}} e^{2 V} Q_{j}+\cdots
$$

At the tree level $K(\Phi, \bar{\Phi})$ is written

$$
K_{t}(\Phi, \bar{\Phi})=-\log (S+\bar{S})+K_{0}(T, \bar{T})
$$

Higher terms are proportional to inverse powers of $(S+\bar{S})$,

$$
K(\Phi, \bar{\Phi})=K_{t}(\Phi, \bar{\Phi})+\frac{1}{8 \pi^{2}} \frac{K_{\bar{i} j}^{(1)}}{S+\bar{S}}+\frac{1}{\left(8 \pi^{2}\right)^{2}} \frac{K_{\bar{i} j}^{(2)}}{(S+\bar{S})^{2}}+\cdots
$$

while the kinetic energy matrix assumes a similar expansion [0], [6]

$$
Z_{\bar{i} j}=Z_{\bar{i} j}^{0}(T, \bar{T})+\frac{1}{8 \pi^{2}} \frac{Z_{\bar{i} j}^{(1)}}{S+\bar{S}}+\frac{1}{\left(8 \pi^{2}\right)^{2}} \frac{Z_{\bar{i} j}^{(2)}}{(S+\bar{S})^{2}}+\cdots
$$

In the above expansions, only the combination $S+\bar{S}$ of the dilaton field appears, as a Peccei-Quinn (PQ) symmetry holds to all orders of perturbation theory.

The superpotential $\mathcal{W}(z)$ is a holomorphic function of the chiral superfields $Q_{i}$ and at the tree level is given by

$$
\mathcal{W}(\Phi, Q)=\frac{1}{3} \lambda_{i j k}(\Phi) Q_{i} Q_{j} Q_{k}+\frac{1}{2} \mu_{i j}(\Phi) Q_{i} Q_{j}+\cdots
$$

where $\{\cdots\}$ stand for possible non-renormalisable contributions. Terms bilinear in the fields $Q_{i}$ refer in fact to an effective higgs mixing term [8, 9]. Perturbative effects may allow dilaton contributions to the superpotential of the form $\propto e^{-8 \pi^{2} S}$, thus breaking the original PQ symmetry which allowed only $S+\bar{S}$ dilaton combinations to appear.

In the following we will assume that the tree level Kähler potential $K_{0}(T, \bar{T})$ can take the following general factorizable form [10, 11]:

$$
K_{0}(T, \bar{T})=-\Sigma_{n} h_{n} \log \left(T_{n}+\bar{T}_{n}\right)
$$

which implies the following form for the gravitino mass

$$
m_{3 / 2}(z, \bar{z})=\frac{|\mathcal{W}(z)|}{\left(\prod_{n}\left(T_{n}+\bar{T}_{n}\right)^{h_{n}}(S+\bar{S})\right)^{1 / 2}}
$$


Now under the modular symmetries, the moduli transform as

$$
T \rightarrow \frac{a T-\imath b}{\imath c T+d}
$$

where $a, b, c, d$ constitute the entries of the $S L(2, \mathbf{Z})$ group elements with $a, b, c, d \in \mathbf{Z}$ and $a d-b c=1$. Next, applying the Kähler transformation,

$$
\begin{aligned}
\mathcal{K} & \rightarrow \mathcal{K}^{\prime}=\mathcal{K}+J(T)+J^{*}(\bar{T}) \\
\mathcal{W} & \rightarrow \mathcal{W}^{\prime}=\mathcal{W} \exp \{-J(T)\}
\end{aligned}
$$

Eqs(17.9) imply that $J(T)$ has the specific form

$$
J(T)=\sum_{n} h_{n} \log \left(\imath c_{n} T_{n}+d_{n}\right)
$$

Following the same procedure, we may obtain the transformation properties of the tree level matrix $Z_{i \bar{j}}^{(0)}(T, \bar{T})$ in the expansion of $\mathrm{Eq}(\overline{0}) \cdot Z_{i \bar{j}}^{(0)}$ is given by the formula 12, 13

$$
Z_{i \bar{j}}^{0}=\delta_{i \bar{j}} \prod_{n}\left(T_{n}+\bar{T}_{n}\right)^{-q_{i}^{n}}
$$

where the exponents are in general rational numbers.

Applying the $\mathrm{SL}(2, \mathbf{Z})$ transformation, Eq(9), to the tree level mass term $Z_{i \bar{j}}^{0} Q_{i} \bar{Q}_{\bar{j}}$ we conclude that the matter fields should transform as follows:

$$
Q_{i} \rightarrow \delta_{i j} Q_{i} \prod_{n} t_{n}^{-q_{i}^{n}}
$$

where, to simplify the subsequent formulae, we have introduced the notation

$$
t_{n}=\imath c_{n} T_{n}+d_{n}
$$

The obtained formulae in Eqs (12,14) may give further restrictions to the transformation properties of the superpotential. Thus, the Kähler transformations Eq(11) and the $J(T)$ form in $\mathrm{Eq}(12)$ imply that the perturbative superpotential is transformed as follows

$$
\mathcal{W} \rightarrow \prod_{n} t_{n}^{-h_{n}} \mathcal{W}
$$

We consider in the following the various terms in the superpotential separately. In fact we are interested in the two types of terms of the perturbative tree level superpotential exhibited in $\mathrm{Eq}\left(\mathrm{6}_{6}\right)$. Thus, the transformation property $\mathrm{Eq}(15)$ together with that of the fields $Q_{i}$ in $\mathrm{Eq}(\sqrt{14})$, imply that the $\mu$ parameter is transformed as follows

$$
\mu_{i j}^{\prime}=\mu_{i j} \prod_{l} t_{l}^{-h_{l}} \prod_{m} t_{m}^{q_{i}^{m}} \prod_{n} t_{n}^{q_{j}^{n}}
$$

A similar expression is expected to hold for the $\lambda_{i j k}$ parameters of the superpotential. 
Thus far, the above procedure gives us no further constraints on the superpotential terms, Yukawa couplings and mass parameters. However, we may impose the constraint that the latter remain invariant under transformations implied by the symmetries of the string. Therefore, we assume the tree level Yukawa couplings and the $\mu$ parameter to be invariant (up to a moduli - independent phase) scalar functions under the action of the modular transformations $\boxplus$. Thus, if we demand invariance of the $\mu$ - term ignoring for simplicity the possible existence of a $T$ - independent phase - we obtain the following relation of modular weights

$$
\prod_{l} t_{l}^{h_{l}}=\prod_{m} t_{m}^{q_{2}^{m}} \prod_{n} t_{n}^{q_{1}^{n}}
$$

A similar reasoning for the case of the Yukawa mass term for the chiral fields $Q_{i}$ leads to the following general condition for the modular weights,

$$
\prod_{l} t_{l}^{h_{l}}=\prod_{m} t_{m}^{q_{i}^{m}} \prod_{n} t_{n}^{q_{j}^{n}} \prod_{r} t_{r}^{q_{r}^{r}}
$$

The Eqs(17,18) are obtained by the simultaneous action of the $\mathrm{SL}(2, \mathrm{Z})$ modular invariance constraints and the invariance of the superpotential parameters of the effective field theory model. In fact they provide specific relations among the modular weights whose role is decisive for the initial conditions of the scalar fields, as can be seen from the form of the tree level scalar matrix $Z_{i \bar{j}}^{0}$.

The parameters of the theory may further be restricted if one imposes the cosmological constant constraint. In order for our procedure to be more transparent, let us simplify the subsequent analysis, assuming that there is a flat direction where $T_{1}=T_{2}=\cdots=T_{N} \equiv T$ which means that the potential depends on a single modulus T. In this case setting $h_{n}=3 / N$ we can simply write, $K_{0}=-3 \log (T+\bar{T})$, to ensure zero vacuum energy. Returning now to the constraint $\mathrm{Eq}(17)$, assuming that this is for a higgs mixing term $\mu H_{1} H_{2}$, we may obtain the following simple relation between the modular weights for the two standard model higgs fields,

$$
q_{1}+q_{2}=h
$$

where $h=N \cdot h_{n}=3$ for the simple example presented here. Furthermore, if the relation $\mathrm{Eq}(18)$ has been obtained, say, for a trilinear coupling of the up-type quark mass matrix $\mathrm{QH}_{2} u^{c}$, a similar constraint may emerge for the corresponding modular weights too, i.e., $q_{Q}+q_{2}+q_{u^{c}}=h$. These constraints can be easily generalised in the case of models with couplings transforming covariantly under the modular symmetries.

\footnotetext{
${ }^{1}$ Actually in $Z_{2} \times Z_{2}$ orbifold construction, the Yukawa couplings are constants, while in Calabi-Yau manifolds they approach a constant value in the large volume limit of the moduli they depend on [6].
} 
Let us see how this type of constraints may give information about the scalar masses at the unification scale. Consider the simple case of untwisted sectors where modular weights are integers. From relation $\mathrm{Eq}(19)$ we may obtain for example $q_{1}=1, q_{2}=2$ or $q_{2}=1, q_{1}=2$ leading to two distinct cases for the initial conditions for the scalar masses $m_{H_{1}}^{2}, m_{H_{2}}^{2}$. In the MSSM for example, such a distinction is welcome; in fact, it implies non - universal boundary conditions for the two higgs doublets which is essential in particular for cases with large $\tan \beta$, i.e., with approximately equal top and bottom Yukawa couplings at the unification scale. Thus, from the two sets of $q_{1,2}$ values above, one may choose the phenomenologically viable case which finally drives the one $(\text { mass })^{2}$ parameter negative at a low scale so that radiative symmetry breaking of the $S U(2) \times U(1)$ occurs. If higgs particles arise from twisted sectors then $q_{1}, q_{2}$ can be any rational number and many solutions can exist even under the apparently restrictive relation $\mathrm{Eq}(19)$.

The above discussion presumes that the $S U(3) \times S U(2) \times U(1)$ symmetry arises at the string scale. It is rather difficult however to obtain correct values of the low energy coupling constants with the ordinary matter fields in the massless spectrum. Indeed, it is well known that using only the MSSM spectrum, unification of the gauge couplings occurs naturally at $M_{U} \sim 10^{16} \mathrm{GeV}$ [1], i.e. almost two orders lower than the string scale. This is rather suggestive for the existence of an IGS. Models with IGS have appeared in a string context [14, 15, 16]. Thus, in the following we wish to extend our previous analysis in these latter cases. In fact, our main motivation for this analysis are string derived models based on $S U(4) \times O(4), S U(5) \times U(1)$ and $S U(3)^{3}$ symmetries. Thus in what follows, we will assume that there is at least one pair of higgs fields, $H_{1,2}$, having the required group properties, and obtaining large vacuum expectation values (vevs) which break the intermediate gauge group down to the MSSM symmetry. For example, in the case of $S U(4) \times O(4) \sim S U(4) \times S U(2)_{L} \times S U(2)_{R}$ these may be $H_{1}=(4,1,2)$ and $H_{2}=(\overline{4}, 1,2)$. In $S U(5) \times U(1)$ these are $5, \overline{5}$ and $10, \overline{10}$. (A particular application will be presented in the subsequent analysis.) We will also perform our computation in the context of our previous simplification, i.e., considering only one modulus $T$ and the dilaton field $S$.

With respect to the fields $z_{I}=\left(z_{S}, z_{0}, z_{1}, z_{2}\right) \equiv\left(S, T, H_{1}, H_{2}\right)$, the scalar potential $V(z)$ is given by

$$
V=e^{G(z)}\left(G_{I} G_{I \bar{J}}^{-1} G_{\bar{J}}-3\right)+|D|^{2}
$$

where $|D|^{2}$ represents the contribution of the D-terms in the potential. Also, with $G_{I}$, we denote the derivatives of $G$ with respect to the fields $z_{I}$, i.e.,

$$
G_{I} \equiv \frac{1}{\mathcal{W}} \mathcal{D}_{I} \mathcal{W}
$$

where $\mathcal{D}_{I} \mathcal{W}=\partial_{I} \mathcal{W}+\mathcal{W} \partial_{I} \mathcal{K}$ is the Kähler derivative. Thus, with respect to the moduli 
$T$, we have for example

$$
(T+\bar{T}) \partial_{T} \mathcal{K}=-h-q_{i} Z_{i}(T, \bar{T}) H_{i} \bar{H}_{i}
$$

and analogously for $\partial_{\bar{T}} \overline{\mathcal{W}}$. In the above basis $z_{I}$, the Kähler metric has the following block diagonal form

$$
G_{I \bar{J}} \equiv \mathcal{K}_{I \bar{J}}=\left(\begin{array}{cc}
\mathcal{K}_{S \bar{S}} & 0 \\
0 & \mathcal{K}_{i \bar{j}}
\end{array}\right)
$$

where the subscripts denote differentiation with respect to the fields $z_{I}$ while $\mathcal{K}_{i \bar{j}}$ is a $3 \otimes 3$ matrix with the indices $i, j$ taking the values $0,1,2$ for the fields $T, H_{1}, H_{2}$ respectively.

In order to calculate the potential $\mathrm{Eq}(20)$, we need the inverse Kähler metric $G_{I \bar{J}}^{-1}$. In particular, $\mathcal{K}_{i \bar{j}}^{-1}$ is given by

$$
\mathcal{K}_{i \bar{j}}^{-1}=\frac{1}{\rho^{2}}\left(\begin{array}{ccc}
1 & \bar{\eta}_{1} & \bar{\eta}_{2} \\
\eta_{1} & \tau^{q_{1}} \rho^{2}+\left|\eta_{1}\right|^{2} & \eta_{1} \bar{\eta}_{2} \\
\eta_{2} & \bar{\eta}_{1} \eta_{2} & \tau^{q_{2}} \rho^{2}+\left|\eta_{2}\right|^{2}
\end{array}\right)
$$

where, we have introduced the convenient notation

$$
\tau^{2} \rho^{2} \equiv Q^{2}=h+q_{1} \frac{H_{1} \bar{H}_{1}}{\tau^{q_{1}}}+q_{2} \frac{H_{2} \bar{H}_{2}}{\tau^{q_{2}}}
$$

with $\tau=T+\bar{T}$, while

$$
\eta_{i}=\frac{q_{i} H_{i}}{T+\bar{T}} \equiv \frac{q_{i} H_{i}}{\tau} \quad, \quad i=1,2
$$

The scalar potential can now be obtained by computing the quantities $G^{I} G_{I} \equiv G^{I} G_{I \bar{J}}^{-1} G^{\bar{J}}$. In oder now to examine in detail the properties of the scalar potential, we need the specific knowledge of the superpotential couplings and in particular that part related to the higgs sector. However, for illustrative purposes let us ignore derivative terms and collect only terms independent of $|\mathcal{W}|^{2}$. In terms of the unrenormalized field vevs $v_{i}=<H_{i}>$ we obtain, at the minimum,

$$
V_{0}=e^{<G>}\left\{3-\left(\frac{h^{2}}{Q^{2}}+\frac{v_{1}^{2}}{\tau^{q_{1}}}+\frac{v_{2}^{2}}{\tau^{q_{2}}}\right)\right\}
$$

where $Q^{2}$ is obtained from $\mathrm{Eq}(25)$ by substituting $v_{i}=<H_{i}>$. Eq(27) determines the two vevs $v_{1,2}$ of the IGS breaking higgs fields. Assuming that the potential at the minimum is zero and equal renormalised vevs (so that the flatness of the effective potential is ensured), we can express the vev as a function of $h$ and the sum $q_{1}+q_{2} \equiv q$. For $h$ close to 3 and the additional constraint $q=3$, we can get a sensible result of vev $\sim 10^{-2} M_{s t r}$. The terms dropped out of the potential could allow for a wider range of $q$ and $h$ giving vev in the desired region. This will be discussed in a future work. 
Up to now, we have analysed how one can determine the higgs vevs which break the IGS and we have expressed their magnitudes as a function of the modular weights. However, we have not yet referred to the mechanism triggering the IGS breaking down to the standard model. In the following, we would like to examine the possibility of breaking the IGS radiatively, pretty much the same way as this happens in the MSSM [17]. Now, the question we would like to ask is if a similar phenomenon may occur in the case of an intermediate symmetry. In a string unified model with IGS, as is the case we are examining here, there are mainly two large scales involved. The first is the string scale $M_{s t r} \sim 5 \times 10^{17} \mathrm{GeV}$, where one is left with a string spectrum having transformation properties under the intermediate gauge group $(S U(4) \times O(4), S U(5) \times$ $U(1)$, etc). The second large scale is the one where the IGS breaks down to the standard model and it is usually assumed to be approximately two orders of magnitude less than the string scale $M_{s t r}$, i.e., it usually coincides with the scale $M_{U}$. The more interesting cases arise in particular IGS models where a much lower intermediate scale is possible, so that negative radiative corrections can grow up enough to turn a higgs $(\text { mass })^{2}$ - parameter negative. This will be the case for the example we will present in the subsequent example.

From the Kähler potential one may obtain through the $Z(T, \bar{T})$ matrix soft mass parameters for the IGS higgs multiplets discussed in detail previously. Their magnitude is controlled by the supersymmetry breaking scale, so their initial values may well be $\mathcal{O}(\leq 1 T e V)$. The question which now arises is whether one of these $(\text { mass })^{2}$ parameters turns negative at the right scale so that the IGS breaks down radiatively. Now, in the case of MSSM, there are two basic ingredients whose role is decisive: $i$ ) the huge gap of the $M_{U}, M_{Z}$ scales which allows radiative corrections to grow up and, ii) the large top-Yukawa coupling. Instead, here we first note that the gap between the two scales $M_{s t r}$ and the conventional supersymmetric unification scale $M_{U}$ is rather short, $M_{s t r} / M_{U} \sim 10^{2}$, and at first sight, it looks rather unlikely that radiative corrections can do the job. Second, a large Yukawa coupling is needed to mimic the role of the top-Yukawa one, in the low energy case. In order to see if the scenario of Radiative Intermediate Symmetry Breaking (RISB) can occur, we will take as an example the $S U(4) \times O(4) \sim S U(4) \times S U(2) \times S U(2)$ model. Here, left and right handed fermions (including the right handed neutrino) are accommodated in the $F=(4,2,1), \bar{F}=$ $(\overline{4}, 1,2)$ representations respectively. The SM symmetry breaking occurs due to the presence of the two standard doublet higgs fields which are found in the $h=(1,2,2)$ representation of the original symmetry of the model (The decomposition of the $h$ under the $S U(3) \times S U(2) \times U(1)$ gauge group is $h(1,2,2) \rightarrow h_{u}\left(1,2, \frac{1}{2}\right)+h_{d}\left(1,2,-\frac{1}{2}\right)$.) The $S U(4) \times S U(2)_{R} \rightarrow S U(3) \times U(1)$ symmetry breaking is realized at a high scale, [18] with the introduction of a higgs pair belonging to $H+\bar{H}=(4,1,2)+(\overline{4}, 1,2)$ representations. Sextets fields $D=(6,1,1)$ appear also in the model. The gauge 
invariant tree level superpotential which is of relevance to our discussion here is [19]

$$
\mathcal{W}=\lambda_{1} F_{L} \bar{F}_{R} h+\lambda_{2} \bar{F}_{R} H \phi_{i}+\lambda_{3} H D D+\lambda_{4} \bar{H} \bar{H} D+\lambda_{5} \phi_{0} h h
$$

From the terms shown in $\mathrm{Eq}(28)$, one can easily figure out that an essential role in the evolution of the soft mass terms of the higgs fields is played by the couplings $\lambda_{3} H H D$ and $\lambda_{4} \bar{H} \bar{H} D$. According to our discussion, these couplings should be chosen sufficiently large if they are supposed to play the role of the top Yukawa coupling in the minimal case. Such a requirement is welcome here since the same couplings determine the masses of the higgs colour triplets living in $D, H, \bar{H}$ which should acquire large masses in order to avoid fast proton decay. In fact, under the SM gauge group the decomposition of the above representations gives $D(6,1,1) \rightarrow D_{3}+\bar{D}_{3}$ and $H(4,1,2) \rightarrow d_{H}+e_{H}+H^{0}$ and similarly for $\bar{H}(\overline{4}, 1,2) \rightarrow \bar{d}_{\bar{H}}+e_{\bar{H}}+\bar{H}^{0}$. Now, down quark type colour triplets will acquire large masses [19] after the symmetry breaking, proportional to $\lambda_{3,4}$, while $e_{H}, e_{\bar{H}}$ will be eaten by the higgs mechanism.

To make clear how the couplings $\lambda_{3,4}$ are involved in the evolution of the soft mass parameters for the neutral higgs components, let us write the corresponding renormalization group equations. We simplify the analysis by ignoring all other Yukawas. We obtain (renaming $H^{0}=H_{1}$ and $\bar{H}^{0}=H_{2}$ ) 18

$$
\begin{aligned}
\frac{d \lambda_{3}}{d t} & =\frac{1}{8 \pi^{2}} \lambda_{3}\left(4 \lambda_{3}^{2}+\lambda_{4}^{2}-\frac{25}{4} g_{4}^{2}-\frac{3}{2} g_{R}^{2}\right) \\
\frac{d \lambda_{4}}{d t} & =\frac{1}{8 \pi^{2}} \lambda_{4}\left(\lambda_{3}^{2}+4 \lambda_{4}^{2}-\frac{25}{4} g_{4}^{2}-\frac{3}{2} g_{R}^{2}\right) \\
\frac{d m_{H_{1}}^{2}}{d t} & =\frac{1}{8 \pi^{2}}\left[\lambda_{3}^{2}\left(6 m_{H_{1}}^{2}+3 m_{D}^{2}\right)-\frac{15}{2} g_{4}^{2} M_{4}^{2}-3 g_{R}^{2} M_{R}^{2}\right] \\
\frac{d m_{H_{2}}^{2}}{d t} & =\frac{1}{8 \pi^{2}}\left[\lambda_{4}^{2}\left(6 m_{H_{2}}^{2}+3 m_{D}^{2}\right)-\frac{15}{2} g_{4}^{2} M_{4}^{2}-3 g_{R}^{2} M_{R}^{2}\right] \\
\frac{d m_{D}^{2}}{d t} & =\frac{1}{8 \pi^{2}}\left[\lambda_{3}^{2}\left(2 m_{D}^{2}+4 m_{H_{1}}^{2}\right)+\lambda_{4}^{2}\left(2 m_{D}^{2}+4 m_{H_{2}}^{2}\right)-10 g_{4}^{2} M_{4}^{2}\right]
\end{aligned}
$$

where $M_{i}$ stands for the gaugino mass of the corresponding group factor. In the following we perform a numerical investigation of the above set of equations in order to find whether it is possible to obtain a negative $(\text { mass })^{2}$. First we determine the initial values of the soft higgs mass parameters from the potential. Taking the derivatives of the potential, the soft masses can in general be of the form [13]

$$
m_{\text {soft }}^{2}=m_{3 / 2}^{2}+V_{0}+\text { modular weight dependent terms }
$$

where $V_{0}$ is essentially the cosmological constant. Thus, in our case, after rescaling to obtain correct normalized fields, while assuming zero cosmological constant, we get

$$
m_{H_{i}}^{2}=m_{3 / 2}^{2}\left(1+q_{i}\right) \quad, \quad i=1,2
$$

${ }^{2}$ In this letter, we will not discuss the origin of supersymmetry breaking. For an attempt in the context of this model see however [20]. 
Obviously, the initial conditions of the two higgs fields depend crucially on the modular weights $q_{1,2}$ which are in general not equal to each other. In the case of untwisted fields, they are integer numbers otherwise they can be any rational number. In any case, according to our assumptions $q_{i}$ 's should satisfy the constraint (19). In order to present an illustrative example, we take $h=3, q_{1}=11 / 4, q_{2}=1 / 4$ and $m_{3 / 2}=100 \mathrm{GeV}$. We assume further, large initial values for the Yukawa couplings $\lambda_{3,4} \sim \mathcal{O}(1)$. In Figure (2a) the two higgs mass - parameters are depicted as a function of the scale $\log _{10} M$. It can be seen that one of them turns negative at a scale $M_{X} \sim$ $2 \times 10^{15} \mathrm{GeV}$, not far from the conventional unification scale $M_{U}$. All other soft squared mass parameters are positive at that scale. In the model under consideration, the IGS breaking scale $M_{X}$ can be even lower, without running into phenomenological troubles as the gauge bosons mediating fast proton decay are absent in this model. Another example is presented in Figure (2b). Here we consider integer modular weights, $q_{1}=2, q_{2}=1$, while again we take $m_{3 / 2}=100 \mathrm{GeV}$. As expected, $m_{H_{2}}^{2}$ is driven now negative at a lower scale. The maximum IGS breaking scale is of course obtained when $q_{1}=3, q_{2}=0$ so that the initial mass parameters have the maximum gap, $m_{H_{2}} / m_{H_{1}}=$ $1 / \sqrt{3}$. For comparison, we show the $m_{H_{2}}$ - plot for the three selective $\left(q_{1}, q_{2}\right)$ pairs in Figure 3. In all the above figures, we choose for convenience to plot $m_{H_{i}}$ 's instead of $m_{H_{i}}^{2}$ parameters. After the scale where $m_{H_{2}}^{2}<0$, we define $m_{H_{2}} \rightarrow-\sqrt{-m_{H_{2}}^{2}}$. From these figures we conclude that the IGS symmetry can break down radiatively naturally, provided that the two modular weights are different in order to create a hierarchy for the two higgs mass parameters at $M_{s t r}$, while the scale $m_{3 / 2}$ should not exceed $(120-130) \mathrm{GeV}$.

In this letter, we have analysed the modular invariance constraints on effective supergravity models with Intermediate Gauge Symmetry which usually arise in four dimensional string constructions. We find that requirements for invariance of Yukawa terms in the superpotential lead to specific relations for the modular weights of the massless spectrum of a particular model. Further constraints for the soft mass parameters are obtained, in particular for the neutral higgs bosons associated with the symmetry breaking. It is found that in particular cases the Intermediate Gauge Symmetry breaks down to the standard model radiatively.

We would like to thank S. Dimopoulos and C. Kounnas for useful suggestions and discussions We also thank CERN - Theory division for kind hospitality during the final stages of this work. 


\section{References}

[1] S. dvips Dimopoulos and H. Georgi, Nucl. Phys. B193(1981)150;

S. Dimopoulos, S. Raby ad F. Wilczek, Phys. Rev. D24(1981)1681;

L.E. Ibáñez and G.G. Ross, Phys. Lett. B105(1982)349;

G. Costa, J. Ellis, G.L. Fogli, D.V. Nanopoulos and F. Zwirner, Nucl. Phys. B297(1988) 244;

J. Ellis, S. Kelley and D.V. Nanopoulos, Phys. Lett. B249 (1990)441; Phys. Lett. B260(1991)131;

P. Langacker and M. Luo, Phys. Rev. D44(1991)817;

H. Arason, D.J. Castaño, B. Keszthelyi, S. Mikaelian, E.J. Piard, P. Ramond and B.D. Wright, Phys. Rev. Lett. 67(1991)2933;

V. Barger, M.S. Berger and P. Ohmann, Phys. Rev. D47(1993)1093;

D.J. Castaño, E.J. Piard and P. Ramond, Phys. Rev. D49(1993)4882;

G.L. Kane, C. Kolda, L. Roszkowski and J.D. Wells, Phys. Rev. D49(1994)6173.

M. Carena, M. Olechowski, S. Pokorski and C.E.M. Wagner, Nucl. Phys. B426(1994)269;

P. Langacker and N. Polonsky, Phys. Rev. D49(1994)1454;

B.C. Allanach and S.F. King, Nucl. Phys. B473(1996)3.

[2] H.P. Nilles, Phys. Rep. 110(1984)1;

H.E. Haber and G.L. Kane, Phys. Rep. 117(1985)75;

A.B. Lahanas and D.V. Nanopoulos, Phys. Rep. 145(1987)1;

S. Ferrara, ed. in 'Supersymmetry', (North Holland, Amsterdam, 1987);

J.L. Lopez, hep-ph/9601208, (sub. in Prog. Part. Nucl. Phys.)

[3] E. Cremmer, S. Ferrara, L. Girardello and A. Van Proyen, Nucl. Phys. B212(1983)413;

J. Bagger, Nucl. Phys. B211(1983)302.

[4] P. Langacker,Phys. Rep. 72(1981)1;

G.G. Ross, Grand Unified Theories, Benjamin Cummings(1985).

[5] S. Ferrara, D. Lüst and S. Theisen, Phys. Lett. B233(1989)147;

S. Ferrara, C. Kounnas, and F. Zwirner,Nucl. Phys. 365(1991)431;

I. Antoniadis, K.S. Narain and T. Taylor, Phys. Lett. B267(1991)37;

S. Kalara, J. Lopez and D.V. Nanopoulos, Phys. Lett. B269(1991)84;

L. Ibañéz and D. Lüst, Nucl. Phys. B382(1992)305;

P. Binetruy and E. Dudas, Nucl. Phys. B442(1995)21.

[6] S. Ferrara, C. Kounnas, and F. Zwirner, Nucl. Phys. B429(1994)589 and references therein. 
[7] V. Kaplunovsky and J. Louis, Nucl. Phys. B444(1995)191.

[8] S.K. Soni and H.A. Weldon Phys. Lett. B126(1983)215;

G. Giudice and A. Masiero, Phys. Lett. B206(1988)480;

V. Kaplunovsky and J. Louis, Phys. Lett. B306(1993)269.

[9] I. Antoniadis, E. Gava, K.S. Narain and T.R. Taylor, Nucl. Phys. 432(1994)187.

[10] E. Witten, Phys. Lett. B155(1985)151.

[11] M. Cvetic, J. Louis and B. Ovrout, Phys. Lett. B206(1988)227;

S. Ferrara and M. Porrati, Phys. Lett. B216(1989)289.

[12] I. Antoniadis, E. Gava and K.S. Narain, Phys. Lett. B 283(1992)209; Nucl. Phys. B383(1992)93.

[13] L. Ibáñez and D. Lüst, Nucl. Phys. B283(1992)305.

[14] H. Kawai, D.C. Lewellen and S.-H.H. Tye, Nucl. Phys. B288(1987)1;

I. Antoniadis, C, Bachas and C. Kounnas, Nucl. Phys. B289(1987)87.

[15] B. Greene, K. Kirklin, P. Miron and G.G. Ross, Nucl. Phys.B278(1986)667;

I. Antoniadis, J. Ellis, J.S. Hagelin and D.V. Nanopoulos, Phys. Lett. B194(1987)23; Phys. Lett. B208(1988)209;

A. Font, L. Ibáñez, F. Quevedo and A. Sierra, Nucl. Phys. B332(1990)421;

A. Faraggi, Phys. Lett. B326(1994)62;

G.K. Leontaris, Phys. Lett. B372(1996)212.

[16] K. Dienes, hep-th/9602045, and references therein (sub. in Phys. Rep.)

[17] K. Inoue, A. Kakuto, H. Komatsu and S. Takeshita, Prog. Theor. Phys. 68(1982) 927 ;

L. E. Ibáǹez and C. Lopez, Phys. Lett. B126(1983)94;

C. Kounnas, A.B. Lahanas, D.V. Nanopoulos and M. Quiros, Phys. Lett. B132 (1983)95; Nucl. Phys. B236(1984)438.

[18] G.K. Leontaris and N.D. Tracas, Phys. Lett. B372(1996)219, hep-ph/9511280;

K. Dienes and A. Faraggi, Nucl. Phys. B467(1996)44, hep-th/9510233;

N. Deshpande, B. Dutta and E. Keith, hep-ph/9604236.

[19] I. Antoniadis and G.K. Leontaris, Phys. Lett. B216(1989)333.

[20] A. Murajama, preprint SH-96-01, (IJMPA, to appear). 


\section{Figure Captions}

Figure 1: Plot of the higgs mass - parameters for $m_{3 / 2}=100 \mathrm{GeV}$, a) $q_{2}=$ $1 / 4, q_{1}=11 / 4$ and b) $q_{2}=1, q_{1}=2$ as a function of the scale $\log _{10} M$. For convenience, - treating properly the negative sign - we show here the $m_{H_{1}}, m_{H_{2}}$, instead of the squared masses.

Figure 2: Plot of the $\mathrm{H}_{2}$-higgs mass - parameter for three $\left(q_{1}, q_{2}\right)$ - pairs and $m_{3 / 2}=116 G e V$. 
Figure 1
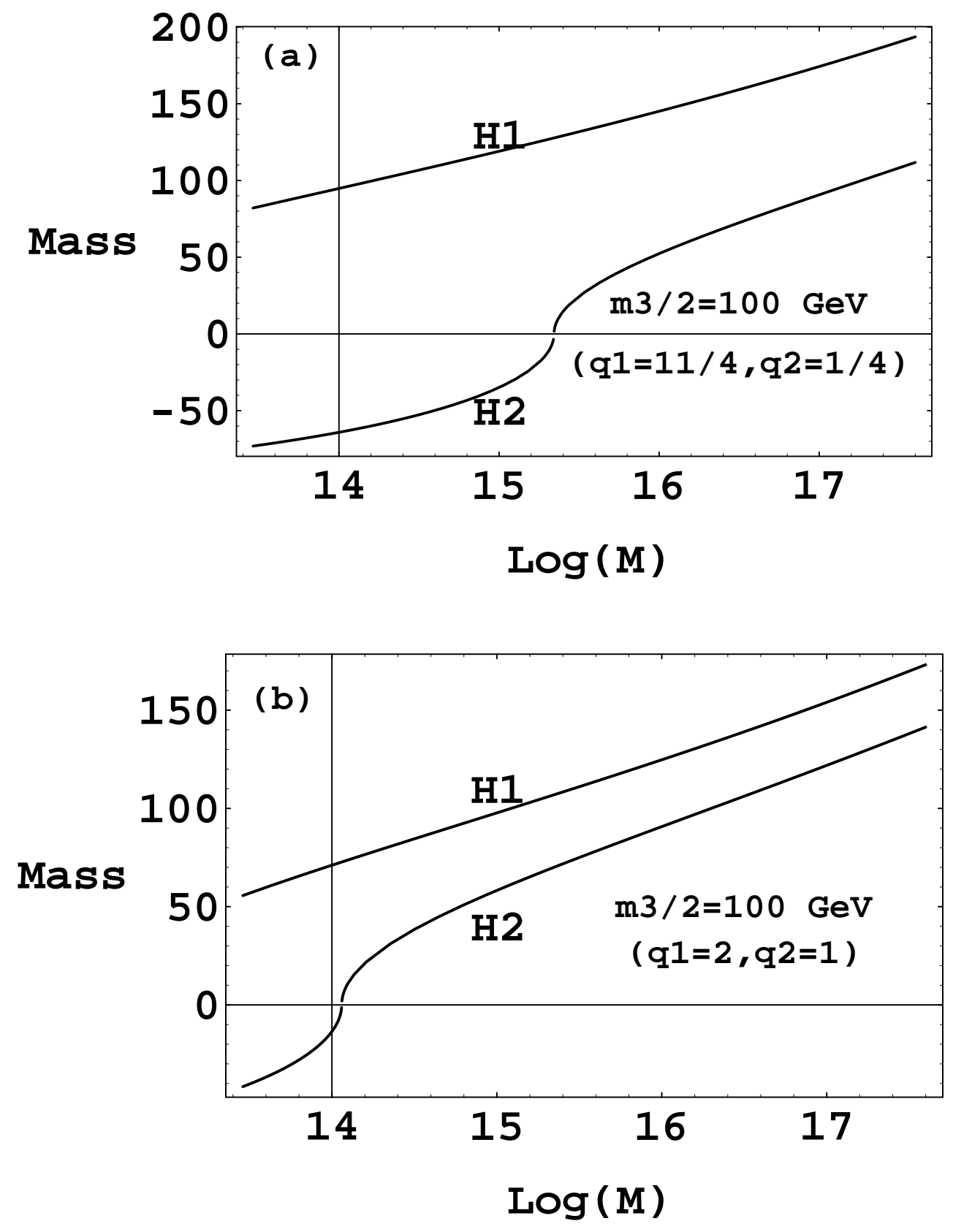
Figure 2

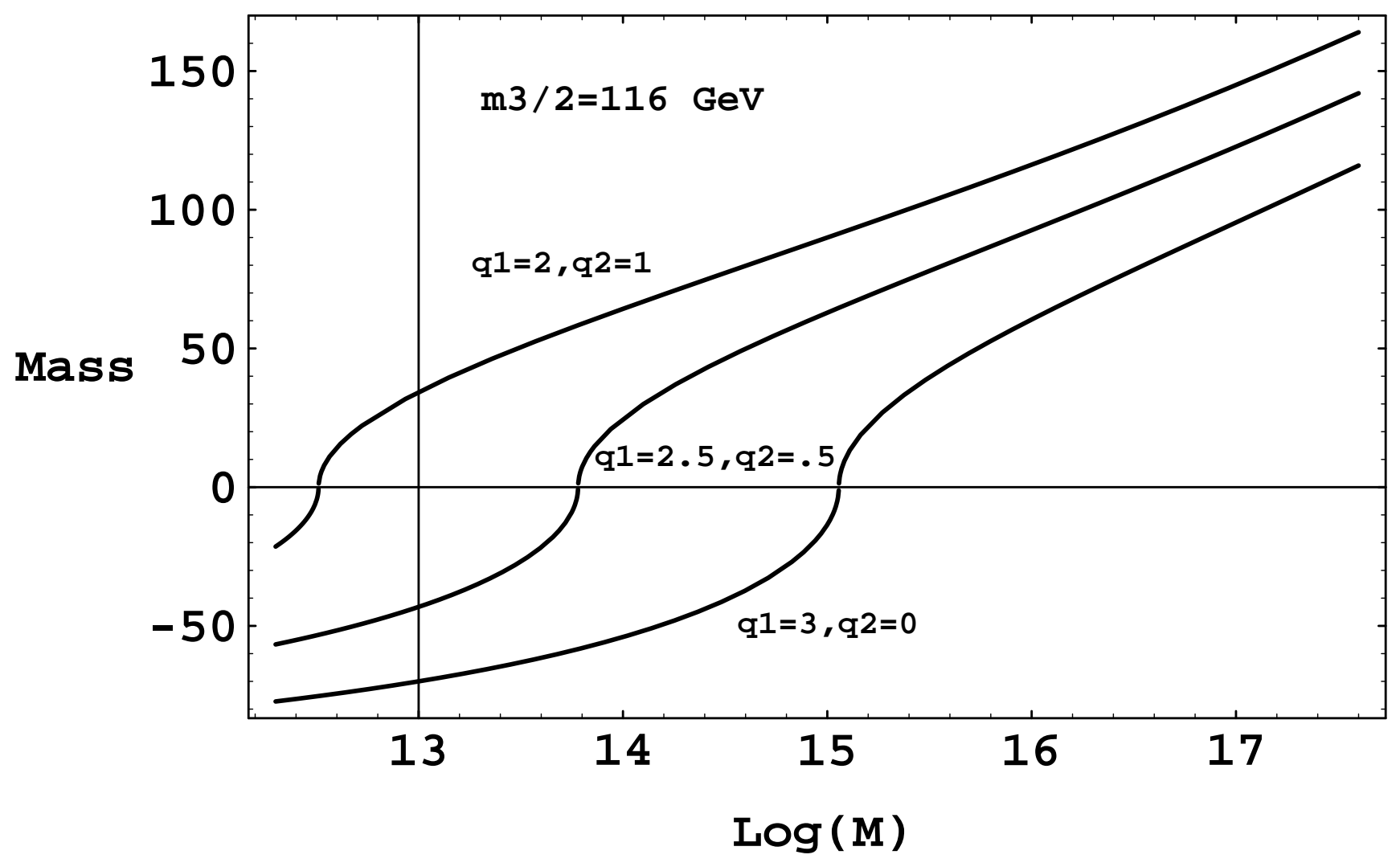

